\title{
DETECTION OF MALARIA PARASITES IN BLOOD SAMPLE USING CASCADING METHOD
}

\author{
M.Brindha \\ Department of BME \\ Velalar College Of Engg.\& Tech, \\ Erode, Tamil Nadu, India
}

\begin{abstract}
Malaria is one of the life threatening disease worldwide. The accurate and timely diagnosis of malaria infection is essential to control and cure the disease. This paper focuses on segmentation and detection of microscopic images infected with malaria parasites using color based cascading method. This method begins with color normalization process followed by gamma correction then noise reduction, exposure compensation, edge compensation, edge enhancement, fuzzy c-means clustering, and morphological process. The results have shown that this method could be successfully used for malaria detection.
\end{abstract}

Keywords-Malaria, Plasmodium, Cascading method, detection, image segmentation

\section{INTRODUCTION}

Malaria is a very serious infectious disease caused by a parasite of the genus Plasmodium which is commonly carried by female Anopheles mosquitoes [1]. According to the World Health Organization statistics, in 2015, it was estimated that the number of malaria cases had decreased to 214 million, and the number of deaths decreased to 438.000.There are many types of plasmodium parasites, but only five species regularly infect humans: plasmodium malariae, plasmodium falciparum, plasmodium knowlesi, plasmodium ovalae, and plasmodium vivax. From the species, p.falciparum is the most lethal [2]. The common way to detect malaria is to draw the patient's blood and then diagnose it using a microscope to determine the infection that occurs based on the colour and morphological changes in the erythrocytes. The process is lengthy, requires expert involvement, and there may be chances for misdiagnosis. Diagnosis using a microscope imagery requires special training and sufficient expertise [5]. This diagnosis has been shown by several studies that manually using microscope is not reliable method if the person doing examination is not an expert or is of inadequate training; especially in rural areas[6][7][8]. In addition different experts can produce different diagnoses and treatments in handling malaria patients [9] [10]. Automatic malaria

\author{
P.Georgia Chris Selwyna \\ Department of BME \\ Velalar College Of Engg. \&Tech, \\ Erode, Tamil Nadu, India
}

\section{PROPOSED ALGORITHM}

The proposed method processes a microscopic blood image. It consists of several steps which include normalization process of RGB, gamma correction, noise reduction, exposure compensation, edge enhancement, fuzzy c-means clustering and ends in morphological processes.

\section{A. RGB Normalization}

RGB Normalization is done to bring all the images to the same brightness level. This process is important since the microscopic blood images we used were acquired by taking photographs of images projected to microscope ocular using mobile phone camera; therefore, the brightness of the images was vastly varied. In normalization process, each pixel value is divided by sum of the pixels value over the channel.

\section{B. Gamma correction}

Gamma correction is a non- linear operation used to encode and decode luminance in still images. Poor contrast is one of the problems in processing microscopic blood images since it can produce erroneous segmentation .we used gamma value of 0.6 to improve the contrast of the images.

\section{Noise Reduction}

Digital images are prone to various types of noise which is the result of errors in the image acquisition process that result in pixel values that do not reflect the true intensities of the real scene. Noise can hamper the segmentation process and need to be removed. We used Gaussian method [20] since it filters the image without damaging its edges.

\section{Exposure Compensation}

Exposure compensation is a technique for adjusting the exposure of the image in the image processing. If the lighting condition is poor, the quality and visibility of images will be severely degraded. The factors that should be considered in exposure compensation include unusual lightning distribution, filters or intended underexposure or over exposure. 


\section{E. Edge Enhancement}

This process is used to sharpen the edges of microscopic images to make plasmodium more prominent compared to its surrounding color. The steps are as follows. First, since the algorithm can only process a single value, the green channel of the image is taken to be processed, or the image is converted into grayscale. Then, the image pixels are processed using $3 \mathrm{x}$ 3 window. The pixels within the window are sorted in ascending order based on their intensity. The next step is to find the median value from the sorted list. After that the median value is used to create two group of pixels: pixels whose intensity value is above (put in the G1 list) and below the median (put in the G2 list). It is then followed by finding the average value of the overall pixel in the $3 \times 3$ matrix, G1 and G2. Finally, the pixel intensity value is determined. Since the processing only done using single channel, the image results are in grayscale.

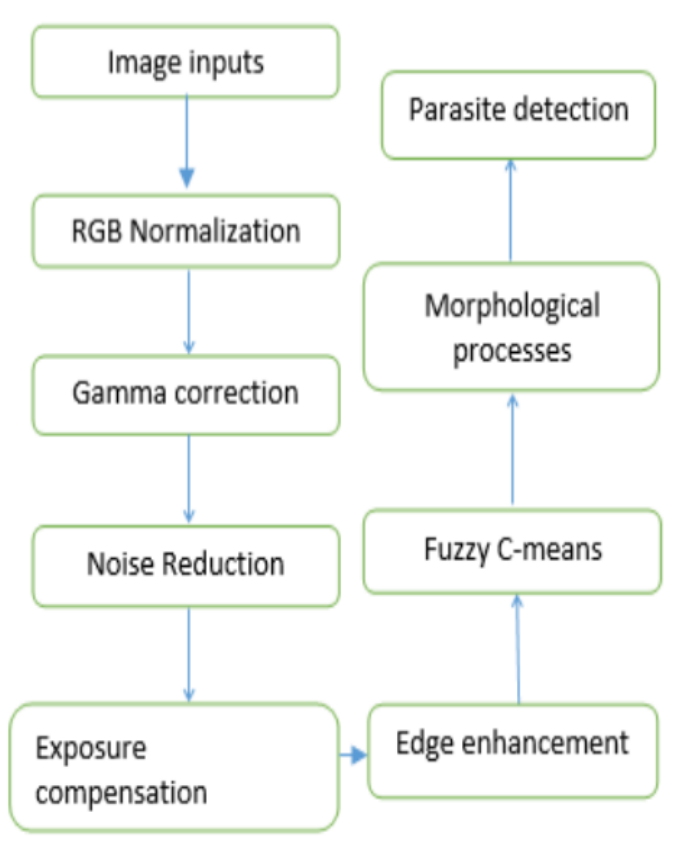

Fig 1. Block Diagram of Cascading method

\section{F. Fuzzy C-means}

Fuzzy C-means is a clustering method that can be used to split object in microscopic images into distinct groups: plasmodium objects are put into the first group whereas blood cells and background color put into the second group.

FCM equation is based on minimizing the value of the objective function. The process is done iteratively by calculating the cluster centroids and then updates the clusters.[21]

\section{G. Morphological Process}

Morphological process is carried out at the last stage to ensure that only the plasmodium is detected. There are two processes used. The first is erosion, aiming to remove all small pixels that are potentially not part of plasmodium. The second process is closing, targeting to close the holes in the plasmodium pixel block.

\section{EXPERIMENT AND RESULT}

In this paper, first we processed the grayscale value of the studied images, then we processed the image green channel only. This occurs from the fifth step: Edge Enhancement. Before this, all the images are processed in RGB color space. The Fuzzy C-means value was calculated for the groups and then used to classify each image of the group. This method produced better results than the conventional methods.
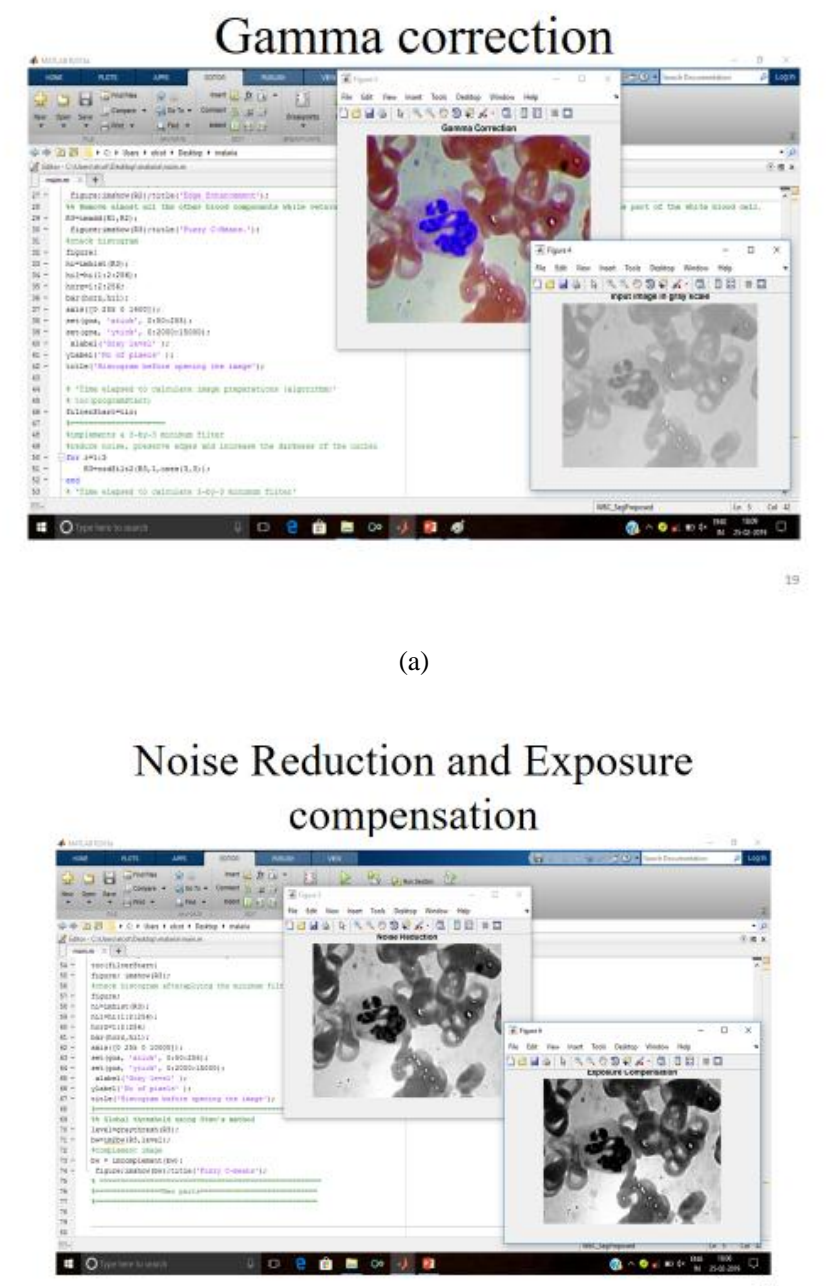

(b) 


\section{International Journal of Engineering Applied Sciences and Technology, 2019 \\ Vol. 4, Issue 2, ISSN No. 2455-2143, Pages 179-181 \\ Published Online June 2019 in IJEAST (http://www.ijeast.com)}

Fig. 2. (a) Gamma Correction (b) Noise Reduction and Exposure Compensenation

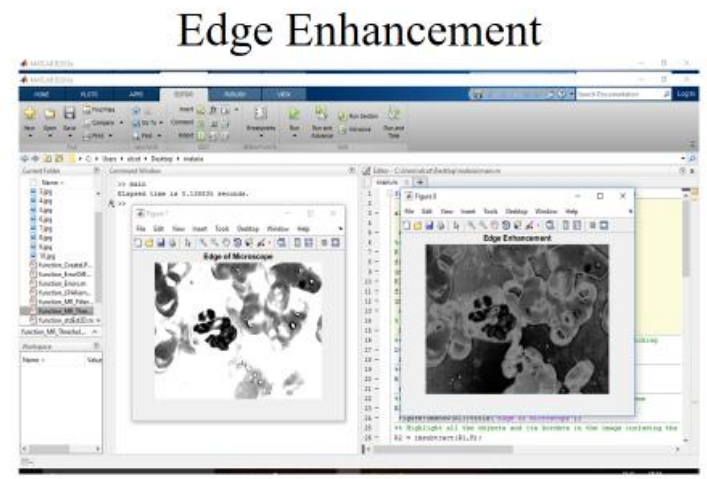

(a)

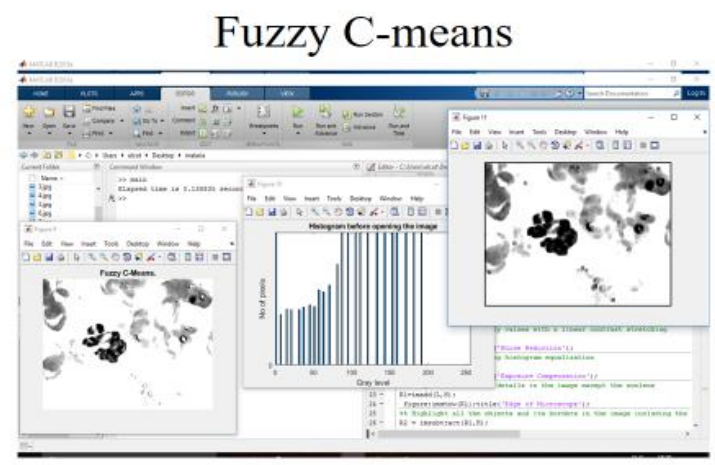

(b)

Fig. 3. (a) Edge Enchancement (b) Fuzzy C-means

\section{CONCLUSION}

The green channel experiment acquired high accuracy results than the grayscale. In our proposed method, we used Fuzzy $\mathrm{C}$-means clustering method for detecting the malarial infection in microscopic images which produced better results than the conventional method.

\section{REFERENCE}

[1] D. A. Ghate and P. C. Jadhav,( 2012) "Automatic Detection of Malaria Parasite from Blood Images," Int. J. Adv. Comput. Technol., vol. 1, no. 3, pp. 66-71.

[2] A. Anand, V. K. Chhaniwal, N. R. Patel, and B. Javidi,( 2012) "Automatic identification of malaria-infected RBC with digital holographic microscopy using correlation algorithms," IEEE Photonics J., vol. 4, no. 5, pp. 14561464.

[3] M. S. Suryawanshi and P. V.V Dixit,(2013) "Comparative Study of Malaria Parasite Detection using Euclidean Distance Classifier \& SVM," vol. 2, no. 11, pp. 29942997.

[4] F.B. Tek, A.G. Dempster, and I. Kale,(2010), "Parasite Detection and Identification for Automated Thin Blood Flim Malaria Diagnosis", Computer Vision and Image Understanding, Vol.114, pp. 21-32.

[5] D. Mas, B. Ferrer, D. Cojoc, S.Finaurini,(2015), "Novel Image Processing to detect Malaria", Optics Communications, Vol.350, pp.13-18.

[6] J. Somasekar and B. Reddy,(2015)“Segmentation of erythrocytes infected with malaria parasites for the diagnosis using microscopy imaging," Comput. Electr. Eng., Vol. 45, pp. 336-351.

[7] C. H. Lai, S. S. Yu, H. Y. Tseng, and M. H. Tsai,(2010) "A protozoan parasite extraction scheme for digital microscopic images," Comput. Med. Imaging Graph., Vol. 34, no.2, pp. 122-130.

[8] Gonzalez, R. C., and Woods, R. E.,(2008), "Digital Image Proceesing”, 3rd ed., Upper Saddle River, New Jersey, Prentice Hall.

[9] N.E. Ross, C. J. Pritchard, D.M. Rubin, and A.G. Duse,(2006) "Automated Image Processing Method for The Diagnosis and Classification of Malaria on Thin Blood Smears ", Medical Biology Engineering Computing, Vol. 44, pp.427-436.

[10] S.W.S. Sio, W. Sun, S.Kumar, W.Z. Bin, S.S.Tan, S.H. Ong, H.Kikuchi, Y. Oshima, and K.S.W. Tan, (2007), "Malaria Count: An Image Analysis-based Program for The Accurate Determination of Parasitemia", Journal of Microbiology Methods, Vo.68, pp. 11-18.

[11] P. Kumswat, Ki. Attakitmongcol and A. Striaew, "A New Approach for Optimization in Image Watermarking by Using Genetic Algorithms", IEEE Transactions on Signal Processing, Vol. 53, No. 12, pp. 4707-4719, December, 2005.

[12] K. Mitiku, G. Mengistu and B. Gelaw,(2003) " The Reliability of Blood Film Examination for Malaria at The Pheripheral Health Unit" ,Ethiopian Journal of Health Development, Vol. 17, pp.197-204. 